\title{
Alerting enhances target identification but does not affect the magnitude of the attentional blink
}

\author{
Thomas M. Spalek • Vincent Di Lollo
}

Published online: 17 November 2010

(C) Psychonomic Society, Inc. 2010

\begin{abstract}
Identification of the second of two targets is impaired when presented less than about $500 \mathrm{~ms}$ after the first. The magnitude of this attentional blink $(\mathrm{AB})$ is known to be modulated by tonic factors (e.g., observer's state of relaxation). The present work examined the effects of a phasic change in observer's state brought about by an alerting stimulus (an aggregate of faint rings) presented in temporal proximity to either letter-target inserted in a temporal stream (RSVP) of digit distractors. In four experiments, identification accuracy of each target was substantially improved by presenting the alerting stimulus either in the target's frame or in the preceding RSVP frame. However, alerting did not modulate the magnitude of the $\mathrm{AB}$. The appearance of an alerting effect on the $\mathrm{AB}$ in Experiment 1 was ascribed to a ceiling effect in Experiment 2. Experiment 3 ruled out endogenous temporal cueing effects; Experiment 4 examined the temporal gradient of alerting. Independence of the alerting and $A B$ effects suggests that the alerting stimuli and the letter targets may be processed along distinct visual pathways.
\end{abstract}

\section{Keywords Attentional Blink $\cdot$ Alerting $\cdot$ Attentional Control}

\section{Introduction}

Attentional limitations in visual information processing are revealed in a phenomenon known as the attentional blink $(\mathrm{AB})$ : when two visual targets are inserted in a stream of

T. M. Spalek $(\bowtie) \cdot$ V. Di Lollo

Department of Psychology, Simon Fraser University,

8888 University Drive,

Burnaby, BC V5A 1S6, Canada

e-mail: tspalek@sfu.ca distractors displayed in rapid serial visual presentation (RSVP), identification accuracy is nearly perfect for the first target (T1) but is dramatically reduced for the second (T2; Raymond, Shapiro, \& Arnell, 1992; Ward, Duncan, \& Shapiro, 1996). To study the temporal course of this T2 deficit, the lag between the two targets is varied systematically in steps of about $100 \mathrm{~ms}$. The deficit is commonly found to be most pronounced when the inter-target lag is short, and to diminish progressively as the lag is increased. The magnitude of the $A B$ is given by the difference between the level of T2 performance at short lags (usually 200 or 300 $\mathrm{ms}$ ) and at the longest lag (usually $700 \mathrm{~ms}$ or longer) which is assumed to lie outside the period of the AB.

$\mathrm{An}$ issue that has received a good deal of attention is whether perception of all attributes of the stimuli presented during the $\mathrm{AB}$ is impaired, or whether some types of information are spared. Among the stimulus attributes known to be impaired are color (Ross \& Jolicœur, 1999), orientation (Kawahara, Di Lollo, \& Enns, 2001), shape (Shapiro, Arnell, \& Drake, 1991), and motion (Kawahara et al., 2001; Krope, Husain, \& Treue, 1998). Among the attributes known to be spared are information regarding one's name (Shapiro, Caldwell, \& Sorensen, 1997), semantic aspects of words (Maki, Frigen, \& Paulson, 1997; Rolke, Heil, Streb, \& Hennighausen, 2001; Shapiro, Driver, Ward, \& Sorensen, 1997; Vogel, Luck, \& Shapiro, 1998), and spatial information conveyed by a cue (Ghorashi, Enns, Klein, \& Di Lollo, 2010; Ghorashi, Klein, \& Di Lollo, 2007; but see Olivers, 2004).

These and similar studies have formed a taxonomic basis for theories of the AB. These studies, however, have been confined to either specific attributes of the stimuli (orientation, motion) or to the tasks performed by the observer (detection, identification). Far fewer investigations have been aimed at correlative analyses of the relationship between the $\mathrm{AB}$ and factors that pertain to the observers themselves rather than - or as well as - the stimuli and the 
tasks. To be sure, there have been studies relating the $\mathrm{AB}$ to ADHD (Mason, Humphreys, \& Kent, 2005), dyslexia (Lum, Conti-Ramsden, \& Lindell, 2007), aging (Maciokas \& Crognale, 2003), and brain injury (Husain, Shapiro, Martin, \& Kennard, 1997), but these studies have been decidedly in the minority, as well as being confined mainly to special populations.

Notably lacking are studies in which the cognitive or attentional state of normal observers was manipulated in order to study how the $\mathrm{AB}$ is affected by factors pertaining to the observer (also known as organismic factors) as distinct from factors external to the observer. An example of such a manipulation has been provided by Olivers and Nieuwenhuis (2005) who found that instructing observers to adopt a more relaxed approach to the task led to an attenuated $\mathrm{AB}$. The instruction to relax induced a change in the state of the observers that presumably lasted for the entire experimental session. In this sense, this manipulation can be said to have had a tonic effect.

In the present study, we asked whether the $\mathrm{AB}$ is also affected by phasic manipulations that cause only a shortlived change in the observer's attentional state. Specifically, the present experiments were designed to find out whether a transient state of alerting triggered by the sudden onset of a task-irrelevant stimulus is impaired during the period of the $\mathrm{AB}$.

Alerting refers to a change in the observer's internal state following the presentation of a transient signal. Such a change in internal state is known to lead to a speeding of the response time (RT) to an ensuing stimulus (Bernstein, Rose, \& Ashe, 1970; Schmidt, Gielen, \& van den Heuvel, 1984). An attentional component akin to alerting was studied by Nakayama and Mackeben (1989) who regarded it as a transient attentional response that facilitates the processing of ensuing stimuli for about $200 \mathrm{~ms}$, and is not subject to voluntary control.

It is important to distinguish between the alerting and the orienting functions of a transient stimulus. Orienting is the selective allocation of attention to a specific location in the visual field or to a specific feature of an upcoming stimulus. In contrast, alerting serves a more diffuse function of enhancing processing over the entire visual field. This difference was recognized early by Hebb (1949) who distinguished between the arousing and the cueing functions of a stimulus. Alerting and orienting functions were decoupled experimentally by Fernandez-Duque and Posner (1997) who proposed that the two are subserved by separate neurophysiological mechanisms. To examine alerting independently of orienting, they displayed stimuli (circle outlines) in several locations simultaneously thus preventing an orienting response to a single location.

In the present work, we employed a conventional $\mathrm{AB}$ paradigm in which two letter targets were inserted in a stream of digit distractors. To study the effects of alerting, one of the frames in the RSVP stream contained an aggregate of faint rings that formed the background on which the digit or letter was displayed (see Fig. 1). Only one set of rings was presented in the RSVP stream, and it appeared at any point in the stream, including the frame preceding $\mathrm{T} 1$ or $\mathrm{T} 2$. The rings were intended to trigger an alerting response and, as shown in Experiment 3, they were not informative as to the occurrence of the targets.

Assessing whether the alerting function is impaired during the period of the $\mathrm{AB}$ can be accomplished by examining performance on $\mathrm{T} 2$ at inter-target lags known to produce an $\mathrm{AB}$. The first requirement is to show that the alerting stimulus does enhance accuracy of T2 identification when presented at an inter-target lag outside the period of the AB. Then, if T2 performance in the alerted and not-alerted conditions is found to be invariant with inter-target lag, it can be concluded that alerting is not impaired during the period of the $\mathrm{AB}$.

\section{Theoretical perspectives}

Based on the evidence that alerting acts to facilitate the processing of ensuing items (Bernstein et al., 1970; Nakayama \& Mackeben, 1989), we now consider predictions from extant theories as to how such facilitation might affect the magnitude of the AB. One way of doing this is to consider the case in which the alerting stimulus (the rings) is presented directly before $\mathrm{T} 1$, thereby facilitating its

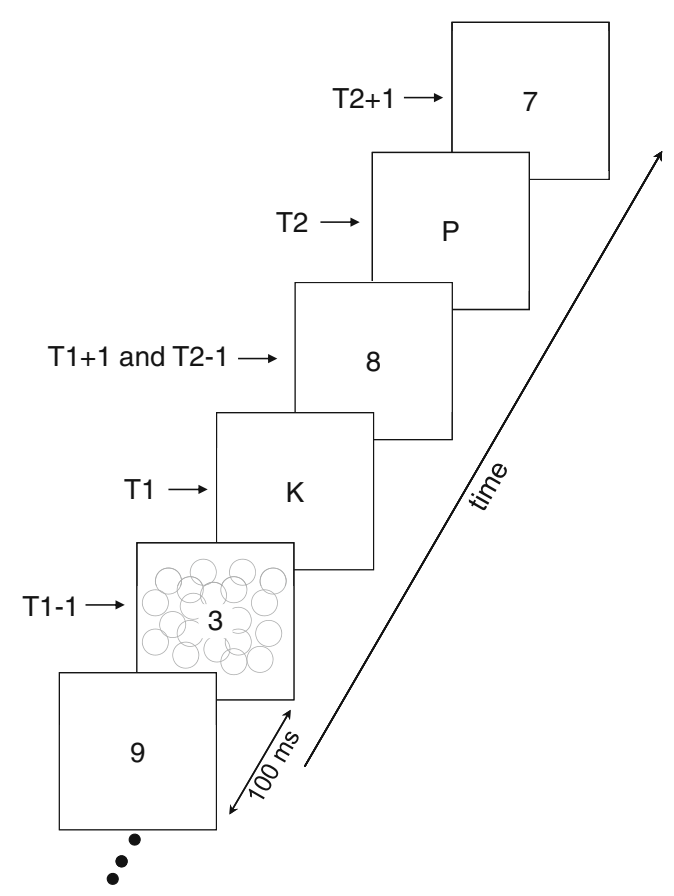

Fig. 1 Experiment 1. Schematic representation of the sequence of events 
processing. Most extant theories predict that facilitation of T1 processing should lead to a corresponding reduction in the $\mathrm{AB}$; one theory predicts the opposite, and one predicts no effect.

Bottleneck theories (Chun \& Potter, 1995; Jolicœur \& Dell'Acqua, 1998) hold that the AB occurs because T2 is vulnerable to masking during a period of delay while the system is busy processing $\mathrm{T} 1$. The probability of $\mathrm{T} 2$ being masked is known to increase directly with the duration of $\mathrm{T} 1$ processing, and hence with the period for which $\mathrm{T} 2$ is vulnerable to masking (Jolicœur \& Dell'Acqua, 1998; Visser, 2007; but see Dux, Asplund, \& Marois, 2008, and response by Olivers, Spalek, Kawahara, \& Di Lollo, 2009). It follows that if the time required to process $\mathrm{T} 1$ is reduced by alerting, the processing delay for $\mathrm{T} 2$ - and hence its vulnerability to masking - will be reduced, with corresponding reduction in the magnitude of the $\mathrm{AB}$.

Resource-depletion theories (Duncan, Ward, \& Shapiro, 1994; Shapiro, Raymond, \& Arnell, 1994) hold that the AB occurs because $\mathrm{T} 1$ takes up resources from a limited supply thereby reducing the resources available for $\mathrm{T} 2$. When resources are depleted, processing of $\mathrm{T} 2$ is slowed down thus extending the period for which $\mathrm{T} 2$ is vulnerable to masking. By speeding up processing of T1, alerting would cause resources to be available sooner for $\mathrm{T} 2$ thus reducing the period for which it is vulnerable to masking. This, in turn, would lead to a reduction in the magnitude of the AB.

Two other theories, Temporary Loss of Control (TLC, Di Lollo, Kawahara, Ghorashi, \& Enns, 2005) and Delayed Attentional Engagement (DAE, Nieuwenstein, Chun, van der Lubbe, \& Hooge, 2005) also predict that enhancing the processing of $\mathrm{T} 1$ through alerting-linked facilitation should reduce the magnitude to the $\mathrm{AB}$. This is because in these theories the $\mathrm{AB}$ is time-locked to the completion of $\mathrm{T} 1$ processing.

The opposite prediction, namely that alerting of $\mathrm{T} 1$ should increase the magnitude of the $\mathrm{AB}$, is made by the Locus Coeruleus theory (Nieuwenhuis, Gilzenrat, Holmes, $\&$ Cohen, 2005). This account is based on the finding that the locus cœruleus, the main component of the noradrenergic system, shows a phasic burst of activity to highly salient stimuli. Such a phasic burst leads to a brief period of increased neural responsivity which benefits the processing of the salient stimulus. The burst of activity is quickly followed by a refractory period of decreased neural responsivity during which perception of temporally trailing stimuli is impaired. By definition, T1 is a salient stimulus that triggers a phasic cycle of activity in the locus coeruleus. Alerting of $\mathrm{T} 1$ should trigger a stronger burst of activity followed by a more pronounced period of reduced responsivity. This would lead to a correspondingly larger $\mathrm{AB}$ at short inter-target lags, when $\mathrm{T} 2$ falls within the period of reduced responsivity.
Finally, no effect of T1 alerting on the magnitude of the $\mathrm{AB}$ is predicted by the Boost-and-Bounce model (Olivers \& Meeter, 2008; see also Olivers, van der Stigchel, \& Hulleman, 2007). In that model, the AB is said to occur when the distractor presented directly after $\mathrm{T} 1$ gains access to high-level processing when it accidentally passes an input filter set to pass only targets. When this happens, the system is said to implement a temporary tightening of input control that causes trailing items-including $\mathrm{T} 2$ at the shorter lags - to be suppressed. As Olivers et al. pointedly note, the tightening of input control is triggered not by $\mathrm{T} 1$ but by the first ensuing distractor. This means that facilitating the processing of $\mathrm{T} 1$ by alerting, as was done in the present work, would not be expected to affect the magnitude of the $\mathrm{AB}$.

The present experiments were designed to achieve four specific objectives: Objective A was to check whether alerting-linked facilitation occurs under the RSVP display conditions commonly used in $\mathrm{AB}$ experiments. Given that Objective A is confirmed, Objective $\mathrm{B}$ was to check whether alerting-linked facilitation of $\mathrm{T} 1$ processing modulates (i.e., attenuates or enhances) the $\mathrm{AB}$, as predicted by the theories outlined above. Objective $\mathrm{C}$ was to check whether the strength of alerting is reduced when the alerting stimulus is presented during the period of the $A B$. Objective $\mathrm{D}$ was to determine whether alerting of $\mathrm{T} 2$ affects the magnitude of the $\mathrm{AB}$ or whether the two effects are independent of one another.

\section{Experiment 1}

Experiment 1 was designed to address all four objectives outlined above. The display sequence consisted of a conventional RSVP stream of white digit distractors and two white letter targets presented on a black background. The alerting stimulus consisted of a random aggregate of 20 small gray rings presented concurrently with the RSVP item in one of six positions in the stream: the distractor preceding $\mathrm{T} 1$ (position $\mathrm{T} 1-1$ ), $\mathrm{T} 1$, the distractor following $\mathrm{T} 1(\mathrm{~T} 1+1)$, the distractor preceding $\mathrm{T} 2(\mathrm{~T} 2-1), \mathrm{T} 2$, or the distractor following $\mathrm{T} 2(\mathrm{~T} 2+1)$.

With reference to the four objectives listed above, Objective A (does alerting enhance target identification?) can be achieved by determining whether T1 performance is higher when $\mathrm{T} 1$ is alerted (conditions $\mathrm{T} 1-1$ and possibly T1) than when it is not alerted (the remaining conditions). Given that Objective A is confirmed, Objective B (does alerting of $\mathrm{T} 1$ modulate the $\mathrm{AB}$ ?) can be achieved by examining whether the magnitude of the $A B$ is less in the T1-alerted conditions than in the remaining conditions. Objectives $\mathrm{C}$ (is alerting reduced during the period of the $\mathrm{AB}$ ?) and $\mathrm{D}$ (does alerting of $\mathrm{T} 2$ modulate the $\mathrm{AB}$ ?) can be 
achieved by examining the interaction in $\mathrm{T} 2$ performance between inter-target lag and whether or not T2 is alerted. The specific comparisons involved in the verification of the four objectives are spelled out below.

\section{Methods}

\section{Observers}

Thirty-one undergraduate volunteers participated for class credit or payment. All reported normal or corrected-tonormal vision and were naïve to the purpose of the experiment.

\section{Apparatus and stimuli}

The stimuli consisted of sequences of digits (0-9) and letters (all upper-case letters of the English alphabet, excepting I, O, Q, and Z) presented in an RSVP stream in the center of a computer monitor. All stimuli were white, subtended approximately $1^{\circ}$ of visual angle, and were presented on a black background (approximately $0.5 \mathrm{~cd} / \mathrm{m}^{2}$ ) at a luminance of $50 \mathrm{~cd} / \mathrm{m}^{2}$ as measured by a Minolta LS 100 luminance meter. Observers sat in a dimly-lit room, and viewed the displays from a distance of approximately $60 \mathrm{~cm}$.

\section{Procedure}

At the beginning of a session, observers were required to read the instructions displayed on the screen, and were invited to ask questions to clarify procedural details. At the beginning of each trial, a small fixation cross was presented in the center of the screen, indicating the location at which the RSVP stream of digits and letters was about to appear. Observers initiated each trial by pressing the space bar. An RSVP stream of digits and letters was displayed directly afterwards. Each item in the RSVP stream remained on the screen for $100 \mathrm{~ms}$, and was replaced immediately by the next item in the stream, yielding a presentation rate of 10 items/s.

The RSVP stream contained a variable number of digit distractors and two letter targets selected randomly without replacement from the letter set described above. The number of distractors preceding $\mathrm{T} 1$ was determined randomly on each trial and varied between 11 and 20. On any given trial, the distractors were selected randomly with replacement from the set of digits $0-9$, with the constraint that the selected digit was not one of the two preceding items. T2 was then presented at one of three lags after the onset of T1: 200, 300, or $700 \mathrm{~ms}$, and was followed by a digit that acted as a mask. Digit distractors continued to be presented during the inter-target interval. Observers were required to ignore the distractors and to report the identity of the two targets by pressing the corresponding keys on the keyboard.

One of the frames in the RSVP stream contained an alerting stimulus in addition to the letter or digit. The alerting stimulus consisted of 20 rings of approximately $1^{\circ}$ diameter and line thickness of approximately $0.05^{\circ}$. The rings had a luminance of $21 \mathrm{~cd} / \mathrm{m}^{2}$ (light gray) and were distributed randomly on each trial within an imaginary rectangle $12^{\circ} \times 8^{\circ}$ centered on fixation. The alerting stimulus could appear in one of the following RSVP frames: $\mathrm{T} 1-1, \mathrm{~T} 1, \mathrm{~T} 1+1, \mathrm{~T} 2-1, \mathrm{~T} 2$, or $\mathrm{T} 2+1$, each appearing an equal number of times at each lag, randomly across trials. A schematic representation of one RSVP sequence is illustrated in Fig. 1. Observers were given 18 practice trials at the beginning of the session. These were followed by a total of 450 trials, 25 for each of the 18 combinations of lag and alerting-stimulus position.

Results and discussion

\section{Alerting effects on $\mathrm{T1}$}

Collapsed across lags, the percentages of correct T1 responses were 95.9, 92.4, 83.2, 82.7, 81.8, and 84.2 for Conditions $\mathrm{T} 1-1, \mathrm{~T} 1, \mathrm{~T} 1+1, \mathrm{~T} 2-1, \mathrm{~T} 2$, and $\mathrm{T} 2+1$, respectively. These data were analyzed in a one-way repeated-measures ANOVA with Rings Position (T1 - 1, $\mathrm{T} 1, \mathrm{~T} 1+1, \mathrm{~T} 2-1, \mathrm{~T} 2$, and $\mathrm{T} 2+1)$ as the factor. The analysis revealed a significant effect of Rings Position, $F(5,150)=$ $53.26, p<.001, \mathrm{MSE}=20.47$. The effect of Ring Position was explored further with a Tukey HSD test which revealed that performance in the $\mathrm{T} 1-1$ condition was higher than in the $\mathrm{T} 1$ condition which was higher than in the remaining four conditions which did not differ from one another.

The results are unambiguous: when the rings were presented directly before - or concurrently with-T1, identification accuracy for $\mathrm{T} 1$ was enhanced, thus revealing an alerting effect. We now consider whether, when presented directly after $\mathrm{T} 1$ (in the $\mathrm{T} 1+1$ position), the rings might have acted as a backward mask, thus impairing T1 identification and producing spurious evidence for $\mathrm{T} 1$ alerting at the $\mathrm{T} 1-1$ and $\mathrm{T} 1$ positions. At least two considerations militate against this option. First, the percentage of correct $\mathrm{T} 1$ identification at the $\mathrm{T} 1+1$ position did not differ significantly from when the rings were presented later in the RSVP stream. Second, the rings were relatively faint and would have made a poor mask even on trials in which they happened to be displayed in proximity to $\mathrm{T} 1$.

\section{Alerting effects on $T 2$}

In this and all subsequent experiments, only those trials in which T1was identified correctly were included for analy- 
sis. This procedure is commonly adopted in $\mathrm{AB}$ experiments on the grounds that, on trials in which T1is identified incorrectly, the source of the error is unknown, and thus its effect on $\mathrm{T} 2$ processing cannot be estimated. The results for $\mathrm{T} 2$ are illustrated in Fig. 2. The T2 data were analyzed in a 3 (Lag: 200, 300, or $700 \mathrm{~ms}$ ) $\times 6$ (Rings Position, T1 -1 , $\mathrm{T} 1, \mathrm{~T} 1+1, \mathrm{~T} 2-1, \mathrm{~T} 2, \mathrm{~T} 2+1)$ repeated-measures ANOVA. The analysis revealed significant effects of Lag, $F(2,60)=84.44, p<.001, \mathrm{MSE}=448.87$, Rings Position, $F(5,150)=66.07, p<.001, \operatorname{MSE}=137.05$, and $\mathrm{a}$ significant interaction effect, $F(10,300)=16.72, p<.001$, MSE $=99.05$. The effect of Ring Position was explored further with a Tukey HSD test which revealed that performance in the $\mathrm{T} 2-1$ and the $\mathrm{T} 2$ conditions did not differ from one another, but both were higher than the remaining four conditions.

Interpretation of the interaction effect revealed in this analysis is complicated by an artifact arising from the experimental design. At Lag 2, the position of the rings in the RSVP stream relative to $\mathrm{T} 2$ was identical in the $\mathrm{T} 1+1$ and in the T2 -1 conditions. Namely, in both conditions, the rings appeared directly before $\mathrm{T} 2$ and, therefore, acted as an alerting signal for it. For this reason, the T2 score at Lag 2 in the $\mathrm{T} 1+1$ condition was artificially high, and may have exaggerated the size of the interaction effect in the above analysis. To check whether the Rings Position $\times$ Lag

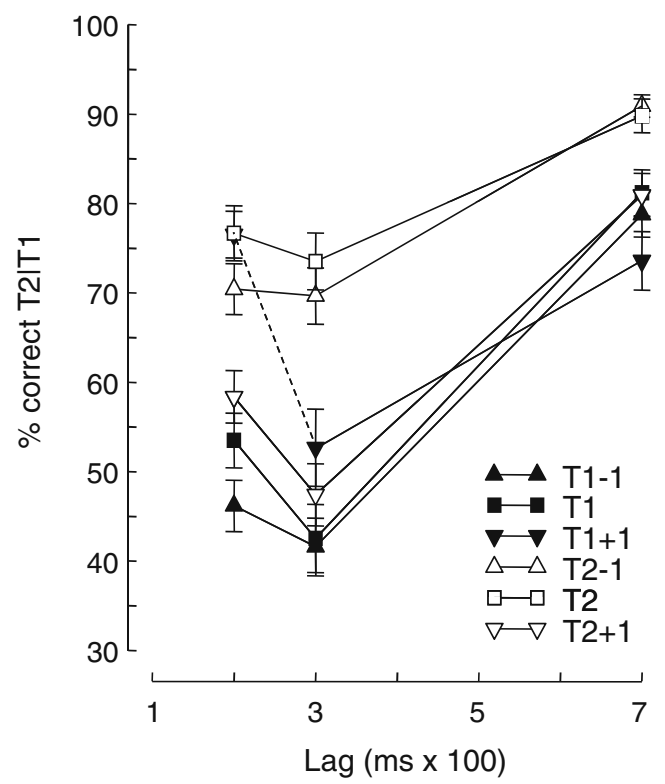

Fig. 2 Experiment 1. Percentage of correct identifications of the second target, given correct identification of the first target. The alerting stimulus was presented either in the frame preceding the first target $(T 1-1)$, or in the same frame as the first target $(T 1)$, or in the frame following the first target $(T 1+1)$, or in the frame preceding the second target $(T 2-1)$, or in the same frame as the second target $(T 2)$, or in the frame following the second target $(T 2+1)$. The segmented line has been added to indicate that Conditions T1 +1 and $\mathrm{T} 2-1$ were, in fact, one and the same condition. See text for explanation interaction remains significant when this artifact is removed, two additional ANOVAs were performed: one on all five conditions but for only Lags 3 and 7, the other on all three lags but with Condition $\mathrm{T} 1+1$ removed. The outcomes of both analyses mirrored the pattern in the original analysis. Notably, the interaction effect was significant in both analyses, $F(5,150)=16.04, p<.001$, MSE $=89.25$, and $F(8,240)=9.51, p<.001$, MSE $=$ 95.93 , respectively. Thus, the interaction effect revealed in the original $\mathrm{T} 2$ analysis remained significant when the procedural artifact was removed. We next examine how these results bear on the four objectives of the present experiment.

Objective A (does alerting-linked facilitation occur under RSVP display conditions?)

Identification accuracy of $\mathrm{T} 1$ provides an affirmative answer to this question. The rings facilitated $\mathrm{T} 1$ performance when they were presented directly before-or simultaneously with-T1. This strongly suggests that the rings acted as an alerting stimulus that led to an improvement in performance analogous to that reported in the alerting literature (Bernstein et al., 1970; Nakayama \& Mackeben, 1989; Schmidt et al., 1984). Notably, the alerting effect was in evidence even though the alerting stimulus was presented within an RSVP stream.

Objective B (does alerting-linked facilitation of T1-processing modulate the AB?)

Having demonstrated that alerting facilitates $\mathrm{T} 1$ performance (Objective A, above), we now consider whether facilitation of T1-processing brought about by alerting of T1 affects the magnitude of the AB. As was noted in the Introduction, the Boost and Bounce model (Olivers \& Meeter, 2008) predicts no effect. The Locus Coeruleus model (Nieuwenhuis et al., 2005) predicts a larger AB. The remaining theories outlined in the Introduction predict that facilitation of $\mathrm{T} 1$ processing should reduce the magnitude of the AB (but see Shapiro, Schmitz, Martens, Hommel, \& Schnitzler, 2006). For this purpose, we combined the six alerting conditions into two groups: the T1-alerted group (T1 - 1 and T1) and the T1-not-alerted group (the remaining four conditions). We then proceeded to examine the magnitude of the $\mathrm{AB}$ in the two groups. After Visser, Bischof, and Di Lollo (1999), we estimated the magnitude of the $\mathrm{AB}$ as the difference in $\mathrm{T} 2$ performance between Lags 3 and 7.

The two sets of averaged scores, illustrated in Fig. 3, were analyzed in a $2(\mathrm{~T} 1$ alerted or not-alerted) $\times 2$ (Lag 3 or Lag 7) repeated-measures ANOVA. The analysis revealed significant effects of Alerting, $F(1,30)=80.47$, 


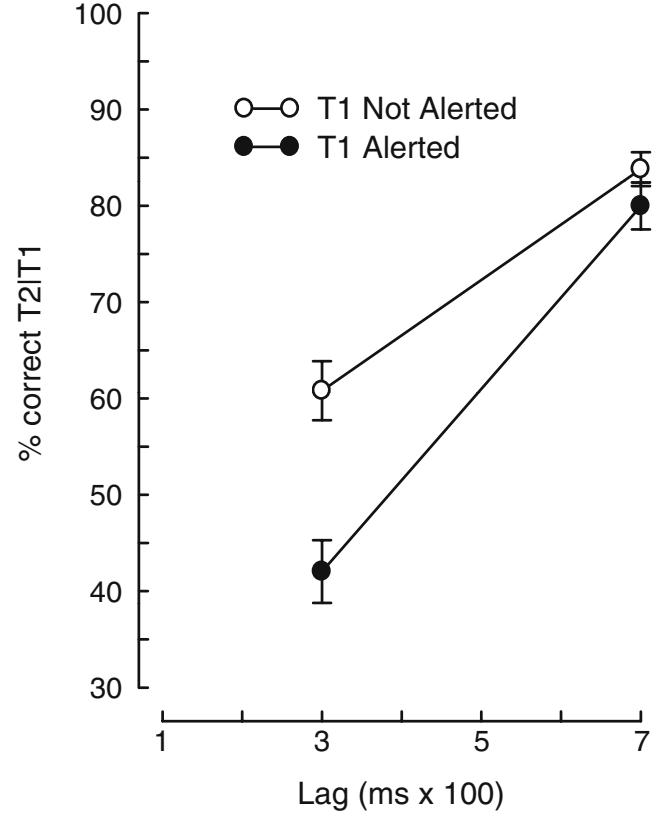

Fig. 3 Experiment 1. Filled symbols percentage of correct secondtarget responses, averaged over Conditions T1 -1 and T1. Open symbols percentage of correct second-target responses, averaged over Conditions T1 $+1, \mathrm{~T} 2-1, \mathrm{~T} 2$, and T2 +1 . See caption of Fig. 2 for definition of abbreviations

$p<.001, \mathrm{MSE}=49.14, \mathrm{Lag}, F(1,30)=157.78, p<.001$, $\mathrm{MSE}=182.34$, and a significant interaction effect, $F(1,30)=$ $54.30, p<.001$, MSE $=31.87$.

As illustrated in Fig. 2, alerting caused T2 accuracy to be considerably higher in Conditions T2 -1 and T2 than in the remaining conditions. It is possible, therefore, that inclusion of the T2 -1 and $\mathrm{T} 2$ conditions in the previous analysis may have biased the outcome. To check on this possibility, we redid the analysis without Conditions T2 - 1 and $\mathrm{T} 2$. The new analysis revealed the same pattern as the earlier one. The effect of T1 Alerting was significant, $F(1,30)=$ $4.22, p=.049, \mathrm{MSE}=51.10$, as was the effect of Lag, $F(1,30)=152.14, p<.001, \mathrm{MSE}=216.19$. Notably, the interaction effect was still significant, $F(1,30)=22.46$, $p<.001, \mathrm{MSE}=39.69$.

The results of these analyses, combined with the graphical evidence in Fig. 3, provide an answer to the question set in Objective $\mathrm{B}$ : the $\mathrm{AB}$ was substantially larger in the T1-alerted conditions than in the T1-not-alerted conditions. This outcome is opposite that predicted by most theories (see Introduction), but is consistent with predictions from the Locus Coeruleus theory.

\section{Objective $\mathrm{C}$ (Is the strength of alerting reduced during} the $A B$ ?)

We answered this question in two steps. First, we checked whether an alerting effect occurred at all in the conditions in which the alerting signal was presented during the period of the AB (Conditions T2 - 1 and T2 at Lag 3). Second, we checked whether the magnitude of the alerting effect in those conditions was less during the period of the $A B$ than outside that period (i.e., at Lag 7). To assess whether an alerting effect occurred at all during the $\mathrm{AB}$, we compared $\mathrm{T} 2$ performance between the alerted and not-alerted conditions at Lag 3. The T2 scores in the T2-alerted conditions (T2 - 1 and T2) were averaged and were compared to the corresponding average of the remaining four (T2-not-alerted) conditions. A $t$ test revealed that mean $\mathrm{T} 2$ performance was significantly higher in the T2-alerted condition $(71.6 \%)$ than in the T2-not-alerted conditions $(46.0 \%), t(30)=$ $10.65, p<.001$. This analysis confirms that an alerting effect on $\mathrm{T} 2$ did occur during the period of the $\mathrm{AB}$.

To assess whether the strength of alerting was reduced during the $\mathrm{AB}$, we compared the size of the alerting effect obtained during the period of the $\mathrm{AB}(\operatorname{Lag} 3)$ with that obtained outside the period of the $\mathrm{AB}$ ( $\operatorname{Lag} 7)$. The $\mathrm{T} 2$ scores in the $\mathrm{T} 2$ alerted conditions (T2 -1 and $\mathrm{T} 2$ ) were averaged, separately for Lags 3 and 7, and were compared with the corresponding averages over the remaining four (T2-not-alerted) conditions, as illustrated in Fig. 4. The data in Fig. 4 were analyzed in a 2 (T2 Alerted or Not-alerted) $\times 2$ (Lag 3 or Lag 7) repeatedmeasures ANOVA which revealed significant effects of Alerting, $F(1,30)=130.85, p<.001, \mathrm{MSE}=82.25$, Lag, $F(1,30)=107.02, p<.001$, MSE $=190.92$, and a significant interaction effect, $F(1,30)=27.70, p<.001, \mathrm{MSE}=53.28$.

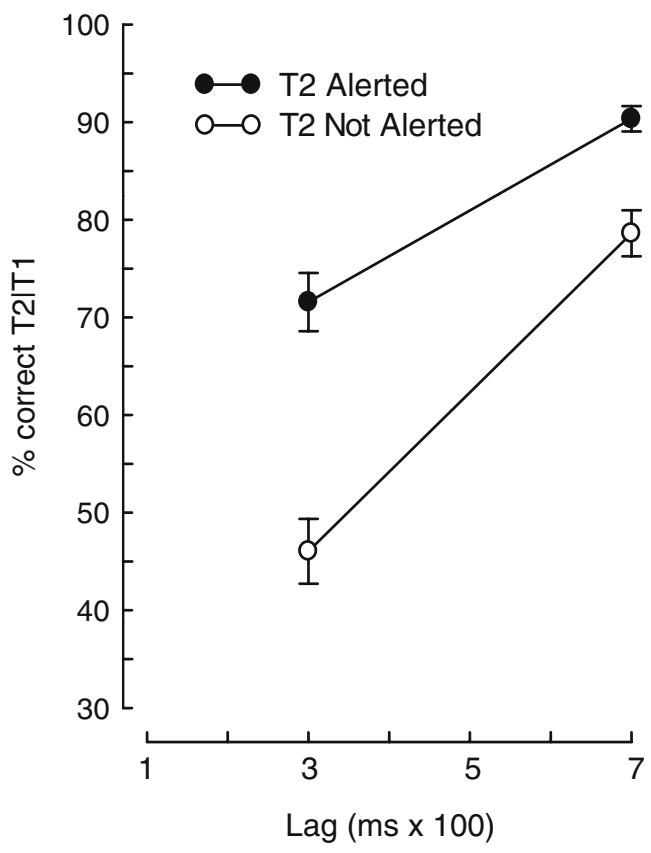

Fig. 4 Experiment 1. Filled symbols percentage of correct secondtarget responses, averaged over Conditions T2 -1 and T2. Open symbols percentage of correct second-target responses, averaged over Conditions $\mathrm{T} 1-1, \mathrm{~T} 1, \mathrm{~T} 1+-1$, and $\mathrm{T} 2+1$. See caption of Fig. 2 for definition of abbreviations 
Based on the outcome of the statistical analysis and the graphical evidence in Fig. 4, the answer to the question implied in Objective $\mathrm{C}$ is in the negative. Namely, the strength of alerting (indexed by the difference T2 Alerted minus $\mathrm{T} 2$ not alerted) is not reduced during the period of the $\mathrm{AB}$. Indeed, the evidence suggests that the strength of alerting is greater during the period of the $\mathrm{AB}$. As can be seen in Fig. 4, the advantage conferred by alerting is greater at $\mathrm{Lag} 3$, when the $\mathrm{AB}$ was maximal, than at $\mathrm{Lag} 7$, which is commonly regarded as lying outside the period of the $\mathrm{AB}$. We hasten to note that this conclusion must be qualified by a possible ceiling effect at Lag 7 in the T2alerted condition (Fig. 4), as noted in the discussion of Objective D, below.

A pattern of results similar to that illustrated in Fig. 4 has been reported by Nieuwenstein et al. (2005), Experiment 2 . Their objective was to find out whether the $A B$ would be attenuated when T2 was precued by two immediately preceding distractors that stood out from all other black distractors because they were colored red - the same as T2 or green. Because of the color match, the red precue served the intended orienting function. This does not mean, however, that the green precue did not also play an orienting role. Being $100 \%$ informative of the onset of T2, both the red and the green precues could reliably orient attention to $\mathrm{T} 2$. But orienting may not have been the only function served by the precues. To wit, the sudden color change associated with the onset of either precue might have served an alerting function, especially since the change occurred within $200 \mathrm{~ms}$ of $\mathrm{T} 2$ onset. Thus, the orienting and the alerting functions were confounded and cannot be separated in the Nieuwenstein at al. study. The results of that study, therefore, are not informative as to the relationship between alerting and the $\mathrm{AB}$, which is the main focus of the present work.

\section{Objective $\mathrm{D}$ (does alerting interact with the $A B$, or are the two effects independent?)}

Defined as the difference in performance between Lags 3 and 7, the magnitude of the $\mathrm{AB}$ appears to be reduced when T2 is alerted. As seen in Fig. 4, the slope of the T2-alerted function is shallower than that of the T2-not-alerted. The difference in slopes is confirmed by the significant interaction effect reported above (Objective $\mathrm{C}$ ). On the face of it, the answer to the question posed in Objective $\mathrm{D}$ is that alerting reduces the magnitude of the $\mathrm{AB}$ and that, therefore, alerting and the $\mathrm{AB}$ are not independent effects.

This conclusion, however, is vitiated by a potential ceiling effect in the Lag 7 scores of the T2-alerted condition (Fig. 4). The reasoning is as follows: suppose that alerting and the $\mathrm{AB}$ are actually independent effects. In that case, the two functions in Fig. 4 should have been parallel instead of converging. Now consider the possibility that alerting and the $\mathrm{AB}$ are independent effects but the Lag 7 scores in the $\mathrm{T} 2$-alerted condition were constrained by the $100 \%$ limit of the response scale (Fig. 4). Such a constraint would cause the two functions to converge at Lag 7. It is possible, therefore, that the two functions converged at Lag 7 not because alerting of $\mathrm{T} 2$ reduced the magnitude of the $\mathrm{AB}$ - causing the $\mathrm{T} 2$-alerted function to be relatively high at Lag 3-but because the response ceiling constrained the T2-alerted function to an artificially low level at Lag 7. Thus, the results of the present experiment must be regarded as inconclusive with respect to Objectives $\mathrm{C}$ and D.

The problem set by a possible ceiling effect can be resolved by using a response measure that, in principle, is unconstrained by ceiling effects. One such measure is reaction time (RT) which was employed in Experiment 2 with the objective of providing an unambiguous answer to the questions posed by Objectives $\mathrm{C}$ and D. The RT measures used in Experiment 2 can yield one of two mutually exclusive outcomes. One is that the interaction effect seen in Fig. 4 is replicated in the RT measure. This would suggest that alerting reduces the magnitude of the $A B$, namely that the alerting effect is stronger during the period of the $\mathrm{AB}$ than beyond it. Alternatively, the RT results may show that alerting is not affected by the $A B$. This would be evidenced by parallel functions for the T2alerted and the T2-not-alerted conditions, and by the absence of an interaction between alerting and lag. Because this alternative is evidenced by a null effect, the number of observers in Experiment 2 was increased to 69 in order to enhance the power of the analysis.

\section{Experiment 2}

\section{Method}

\section{Observers}

Sixty-nine undergraduate volunteers participated for class credit or payment. All reported normal or corrected-tonormal vision and were naïve to the purpose of the experiment.

\section{Apparatus, stimuli, and procedures}

These were the same as in Experiment 1 except for the following. $\mathrm{T} 2$ was either the letter $\mathrm{C}$ or $\mathrm{G}$, and was followed by the \& symbol. The observers were instructed to indicate whether $\mathrm{T} 2$ was $\mathrm{C}$ or $\mathrm{G}$ as quickly as possible by entering it on the keyboard. They then entered the T1 response at their leisure. This permitted T2 RTs to be recorded in addition to accuracy of both responses. In order to decrease the length of the experimental session, the 
number of conditions was reduced to five by omitting the $\mathrm{T} 2+1$ condition.

Results and discussion

\section{Alerting effects on $\mathrm{T1}$}

Collapsed across lags, the percentages of correct $\mathrm{T} 1$ responses were 90.0, 86.6, 76.3, 76.5, and 75.0 for Conditions $\mathrm{T} 1-1, \mathrm{~T} 1, \mathrm{~T} 1+1, \mathrm{~T} 2-1$, and $\mathrm{T} 2$, respectively. These data were analyzed in a one-way repeated-measures ANOVA with Rings Position (T1 - 1, $\mathrm{T} 1, \mathrm{~T} 1+1, \mathrm{~T} 2-1$, and $\mathrm{T} 2$ ) as the factor. The analysis revealed a significant effect of Rings Position, $F(4,272)=$ 92.35, $p<.001, \mathrm{MSE}=106.69$. The effect of Ring Position was explored further with a Tukey HSD test which revealed that performance in the $\mathrm{T} 1-1$ condition did not differ from that in the $\mathrm{T} 1$ condition, but both were higher than the remaining three conditions which did not differ from one another.

\section{Alerting effects on $T 2$}

The results for T2 are illustrated in Fig. 5, separately for RT and accuracy. The accuracy scores (Fig. 5b) were analyzed in a 3 (Lag: 200, 300, or $700 \mathrm{~ms}$ ) $\times 5$ (Rings Position, T1 $1, \mathrm{~T} 1, \mathrm{~T} 1+1, \mathrm{~T} 2-1, \mathrm{~T} 2)$ repeated-measures ANOVA. The analysis revealed significant effects of Lag, $F(2,136)=$ $131.27, p<.001, \mathrm{MSE}=176.71$, Rings Position, $F(4,272)=$ $69.48, p<.001, \mathrm{MSE}=104.51$, and a significant interaction effect, $F(8,544)=11.71, p<.001$, MSE $=92.41$. The outcome of this analysis mirrors the outcome of the corresponding analysis in Experiment 1.

The RT data for T2 (Fig. 5a) were analyzed in a 3 (Lag: $200,300$, or $700 \mathrm{~ms}) \times 5$ (Rings Position, T1 $-1, \mathrm{~T} 1, \mathrm{~T} 1+$ $1, \mathrm{~T} 2-1, \mathrm{~T} 2)$ repeated-measures ANOVA. The analysis revealed significant effects of Lag, $F(2,136)=109.70, p<$ .001 , MSE $=33,105.11$, and Rings Position, $F(4,272)=$ $16.20, p<.001, \mathrm{MSE}=17,154.14$, but the interaction effect was not significant, $F(8,544)=1.30, p=.24$, MSE $=$ $17,235.74$.

The absence of a significant interaction effect means that the magnitude of alerting did not vary across conditions as a function of lag. This is the result of principal interest in the present context because it indicates that the significant interaction effect obtained in the corresponding analysis in Experiment 1, as well as in the accuracy results in the present experiment (Fig. 5b) were probably due to ceiling effects. The interaction effect obtained in Experiment 1 and in the accuracy measure in the present experiment vanish when the constraint imposed by the ceiling of the response scale is removed by using RT instead of accuracy as the response measure.
Although the interaction between Ring Position and Lag was not significant, it is worth stressing that a good deal of the variance in that interaction was probably attributable to the $\mathrm{T} 1+1$ condition. As we noted in Experiment 1, at Lag 2 the position of the rings in the RSVP stream relative to T2 was identical in the $\mathrm{T} 1+1$ and in the $\mathrm{T} 2-1$ conditions. Namely, in both conditions the rings appeared directly before $\mathrm{T} 2$ and, therefore, acted as an alerting signal for it. This caused the T2 RT at Lag 2 in the T1 +1 condition to be artificially low, thus exaggerating the size of the interaction effect. For this reason, the analysis was redone omitting the $\mathrm{T} 1+1$ condition. The reanalysis revealed significant effects of Lag, $F(2,136)=97.03, p<.001$, MSE $=31712.13$, and Rings Position, $F(3,204)=18.76$, $p<.001, \mathrm{MSE}=16,536.31$, with a nonsignificant interaction effect, $F(6,408)=0.36, p=.904$, MSE $=17,502.81$. The critical aspect of this reanalysis is that the $F$ ratio for the interaction effect was less than unity. This is important because, assuming that the variance estimate based on the present 69 observers is dependable, the interaction effect would not reach significance even if the number of observers were increased manyfold. This consideration is consistent with the inference that alerting and the $\mathrm{AB}$ are independent effects, and that the interaction effect obtained in the accuracy scores was due to a ceiling effect.

In light of these results, we can now provide a more definitive answer to the questions posited in Objectives $\mathrm{C}$ (Is the strength of alerting reduced during the $A B$ ?) and $\mathrm{D}$ (does alerting interact with the $A B$, or are the two effects independent?). On the strength of the RT results (Fig. 5a), it can be concluded that the strength of alerting is not reduced during the AB. Similarly, it can be concluded, at least provisionally, that alerting and the $\mathrm{AB}$ are independent effects. ${ }^{1}$

\section{Experiment 3}

In Experiment 1, the alerting stimulus was always presented in close temporal contiguity with a target. This contingency

\footnotetext{
${ }^{1}$ It could be argued that a task-switch was present in Experiment 2 but not in Experiment 1. Namely, in Experiment 2, the response was speeded for $\mathrm{T} 2$ but not for $\mathrm{T} 1$, and $\mathrm{T} 1$ was selected from a set of 20 letters whereas T2 was selected from a set of only 2 letters. It could further be argued that the $\mathrm{AB}$ obtained with task switching and that obtained without task switching might be mediated by different mechanisms. In that case, the finding in Experiment 2 that alerting and the $\mathrm{AB}$ are independent effects would apply to $\mathrm{ABs}$ produced by a task switch but not to ABs produced by mechanisms other than task switching. This interpretation, however, is inconsistent with the bulk of the $\mathrm{AB}$ literature in which the $\mathrm{ABs}$ obtained with the procedures employed in Experiment 2 have been interpreted within the same conceptual frameworks - and as relevant to the same theories-as those obtained with more conventional procedures (e.g., Jolicœur \& Dell'Acqua, 1998; Visser, 2007).
} 
Fig. 5 Experiment 2. a Mean median reaction times to the second target, given correct identification of both targets. b Percentage of correct identifications of the second target, given correct identification of the first target. See caption of Fig. 2 for definition of abbreviations in the legend

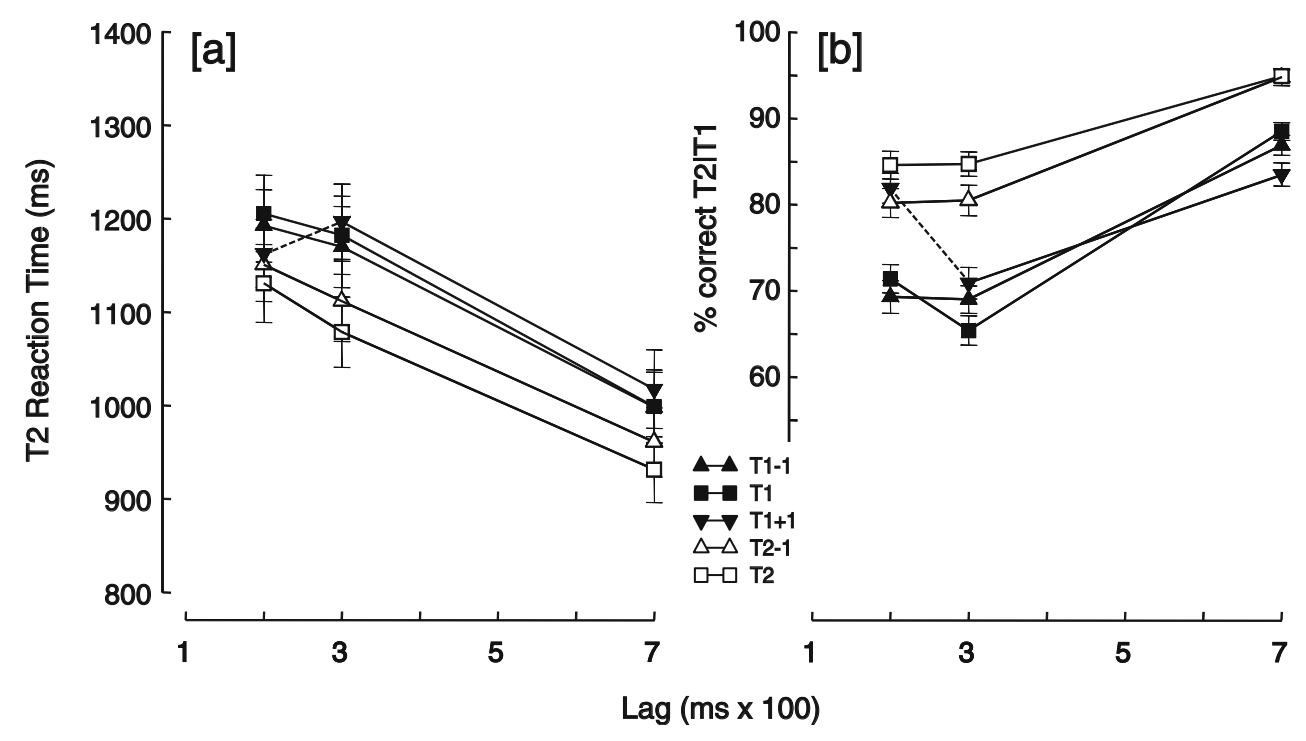

might have led the observers to try to use the alerting stimulus as a predictor of target occurrence. In this case, the higher levels of performance seen in Experiment 1 when T1 and T2 were alerted could be ascribed to an endogenous temporal orienting response rather than - or in addition toan exogenous alerting response.

Ideally, to be completely unpredictive, the alerting stimulus should appear with equal likelihood in each frame in the RSVP stream. This, however, would require the total number of experimental trials to be increased beyond 2,000 and the experimental session to be extended to over $4 \mathrm{~h}$. Because it would be unrealistic to expect observers to remain focused on the task for such a long period, we opted to simply decrease the contingency relative to Experiment 1. If predictiveness was a factor in Experiment 1, reducing the predictiveness of the alerting stimulus should result in a corresponding reduction in the alerting effect. This possibility was examined and rejected in Experiment 3, in which the predictiveness of the alerting stimulus was reduced by presenting it in contiguity with a target on only $1 / 3$ of the trials.

\section{Method}

\section{Observers}

Thirty undergraduate volunteers participated for class credit or payment. All reported normal or corrected-to-normal vision and were naïve to the purpose of the experiment.

\section{Apparatus, stimuli, and procedures}

These were the same as in Experiment 1 except that the rings were presented in one of the following RSVP positions: $\mathrm{T} 1-6, \mathrm{~T} 1-5, \mathrm{~T} 1-1, \mathrm{~T} 2-1, \mathrm{~T} 2+2$, or $\mathrm{T} 2+3$.
Results and discussion

\section{Alerting effects on $\mathrm{Tl}$}

Collapsed across lags, the percentages of correct T1 responses were $81.9,81.9,95.9,80.9,83.7$, and 85.4 for Conditions $\mathrm{T} 1-6, \mathrm{~T} 1-5, \mathrm{~T} 1-1, \mathrm{~T} 2-1, \mathrm{~T} 2+2$, and $\mathrm{T} 2+3$, respectively. These data were analyzed in a one-way repeatedmeasures ANOVA with Rings Position (T1 - 6, T1 - 5, T1 $1, \mathrm{~T} 2-1, \mathrm{~T} 2+2$, and $\mathrm{T} 2+3$ ) as the factor. The analysis revealed a significant effect of Rings Position, $F(5,145)=$ 40.84, $p<.001, \mathrm{MSE}=22.85$. As was the case in Experiment 1, the effect of alerting was observed in Condition T1 - 1 where accuracy of T1 identification was above that in all other conditions.

\section{Alerting effects on $T 2$}

The results for T2 are illustrated in Fig. 6. The T2 data were analyzed in a 3 (Lag: 200, 300, or $700 \mathrm{~ms}$ ) $\times 6$ (Rings Position, T1 $-6, \mathrm{~T} 1-5, \mathrm{~T} 1-1$, T2 -1 , T2 +2 , or $\mathrm{T} 2+3$ ) repeated-measures ANOVA. The analysis revealed significant effects of Lag, $F(2,58)=100.92, p<.001, \mathrm{MSE}=532.93$, Rings Position, $F(5,145)=66.70, p<.001, \mathrm{MSE}=121.10$, and a significant interaction effect, $F(10,290)=6.36, p<$ $.001, \mathrm{MSE}=97.13$. The effect of Ring Position was explored further with a Tukey HSD test which revealed that performance in the T2-1 was higher than in the remaining five conditions which did not differ from one another.

The principal objective of Experiment 3 was to examine whether performance in Experiment 1 might have been boosted by the alerting stimulus acting as an endogenous cue. To this end, we compared performance in the T2 - 1 condition in Experiments 1 and 3. To the extent that the alerting stimulus had acquired a predictive value, the level 


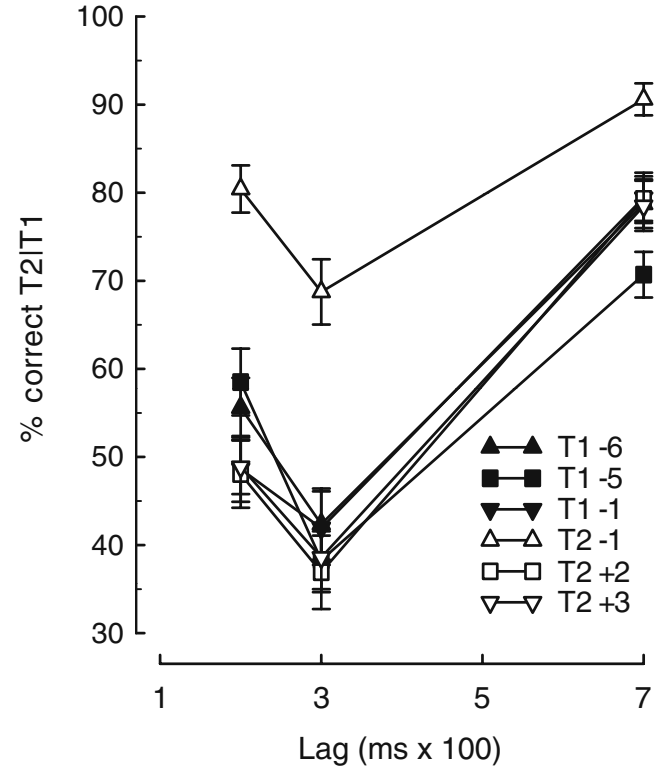

Fig. 6 Experiment 3. Percentage of correct identifications of the second target, given correct identification of the first target. The alerting stimulus was presented either six frames before the first target $(T 1-6)$, or five frames before the first target $(T 1-5)$, or in the frame preceding the first target $(T 1-1)$, or in the frame preceding the second target $(T 2-1)$, or two frames following the second target $(T 2+$ $2)$, or three frames following the second target $(T 2+3)$

of performance in Experiment 1 should exceed that in Experiment 3. The data from the two experiments were analyzed in a 2 (Experiment: 1 or 3 ) $\times 3$ (Lag: 200 , 300, or $700 \mathrm{~ms})$ mixed-design ANOVA. The analysis revealed a significant effect of Lag, $F(2,118)=56.70, p<.001$, MSE $=132.67$. The effect of Experiment was not significant, $F(1,59)=2.16, p=.15, \mathrm{MSE}=301.04$. This strongly suggests that predictiveness was not an important determinant of performance in Experiment 1. The analysis also revealed a marginally significant interaction effect, $F(2,118)=3.02, p=.052, \operatorname{MSE}=132.67$. It must be noted, however, that the interaction was due exclusively to lower performance at Lag 2 in Experiment 1, which is the opposite of what should be expected if the observers were using the alerting stimulus as a predictive cue.

\section{Experiment 4}

Experiment 4 was designed to examine whether the strength of the alerting effect varies in a graded manner as a function of the temporal separation between the alerting stimulus and the trailing target. The results of the previous experiments showed that presenting the alerting stimulus $100 \mathrm{~ms}$ before the target was more effective than presenting it at the same time as the target. What is not known is whether the alerting function peaked at an SOA of $100 \mathrm{~ms}$ or at a longer SOA. A broader range of SOAs was examined in Experiment 4 by presenting the alerting stimulus either 100,200 , or $300 \mathrm{~ms}$ before $\mathrm{T} 1$ or $\mathrm{T} 2$.

Method

\section{Observers}

Thirty-three undergraduate volunteers participated for class credit or payment. All reported normal or corrected-to-normal vision and were naïve to the purpose of the experiment.

\section{Apparatus, stimuli, and procedures}

These were the same as in Experiment 1 except that the rings were presented in one of the following RSVP positions: $\mathrm{T} 1-3, \mathrm{~T} 1-2, \mathrm{~T} 1-1, \mathrm{~T} 2-3, \mathrm{~T} 2-2$, or $\mathrm{T} 2-1$. A schematic illustration of the sequence of events is presented in Fig. 7, separately for the three lags.

Results and discussion

\section{Alerting effects on $T 1$}

Collapsed across lags, the percentages of correct T1 responses were $82.1,90.8,96.5,89.4,82.5$, and 76.6 for

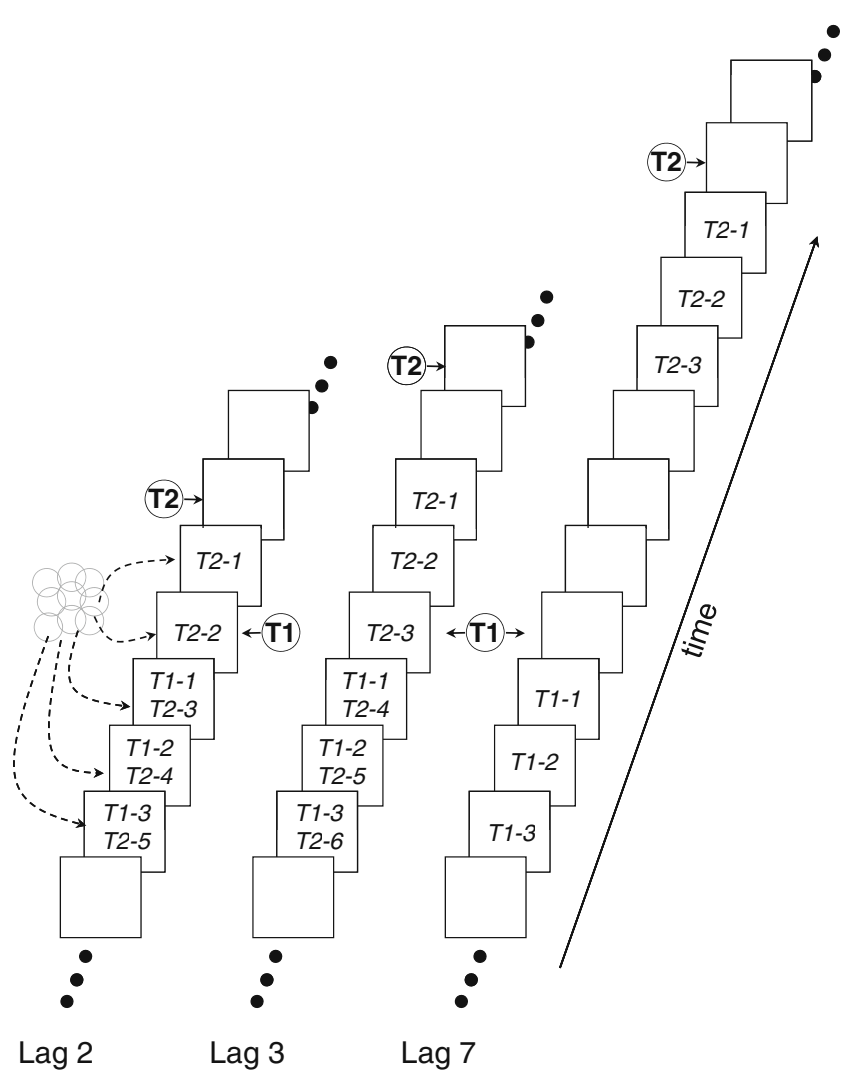

Fig. 7 Experiment 4. Schematic representation of the sequence of events 
Conditions $\mathrm{T} 1-3, \mathrm{~T} 1-2, \mathrm{~T} 1-1, \mathrm{~T} 2-3, \mathrm{~T} 2-2$, and $\mathrm{T} 2-$ 1 , respectively. These data were analyzed in a one-way repeated-measures ANOVA with Rings Position (T1 - 3, $\mathrm{T} 1-2, \mathrm{~T} 1-1, \mathrm{~T} 2-3, \mathrm{~T} 2-2$, and $\mathrm{T} 2-1)$ as the factor. The analysis revealed a significant effect of Rings Position, $F(5,160)=36.68, p<.001, \mathrm{MSE}=46.73$. The effect of Ring Position was explored further with a Tukey HSD test which revealed that each condition differed from each other condition, except for the following pairs which did not differ from one another: $\mathrm{T} 1-3 / \mathrm{T} 2-2$ and $\mathrm{T} 1-2$ / T2 - 3 .

The results of the present experiment are consistent with the conclusion that the maximal alerting effect is observed when the alerting stimulus is presented $100 \mathrm{~ms}$ before the target. That is, the strength of alerting increased progressively in Conditions $\mathrm{T} 1-3, \mathrm{~T} 1-2$, and $\mathrm{T} 1-1$. On the face of it, the finding that there was also a progressive decrement in the strength of alerting in Conditions T2 - 3, $\mathrm{T} 2-2$, and $\mathrm{T} 2-1$ might seem inconsistent with the previous findings that alerting of $\mathrm{T} 2$ had no effect on $\mathrm{T} 1$ performance.

In fact, this can be understood if it is realized that, as seen in Fig. 7, the location of the alerting stimulus in the RSVP stream did not vary independently with respect to T1 and T2. For example, at Lag 2, presenting the alerting stimulus at the $\mathrm{T} 2-3$ position coincided with the $\mathrm{T} 1-1$ position. Hence, T1 performance at the T2 -3 position was spuriously boosted by the presence of the alerting stimulus in the immediately preceding frame. In general, T1 performance was boosted in those conditions in which the alerting stimulus was presented at the same time as $\mathrm{T} 1$ or shortly before it. This was the case in Condition T2 - 3 at Lags 2 and 3, and in Condition T2 - 2 at Lag 2. From this perspective, there is no inconsistency between the patterns of T1-alerting obtained in the present experiment and in the previous experiments.

\section{Alerting effects on $T 2$}

The results for $\mathrm{T} 2$ are illustrated in Fig. 8. The T2 data were analyzed in a 3 (Lag: 200, 300, or $700 \mathrm{~ms}$ ) $\times 6$ (Rings Position, T1 - 3, T1 - 2, T1 - 1, T2 - 3, T2 - 2, and T2 1) repeated-measures ANOVA. The analysis revealed significant effects of Lag, $F(2,64)=143.71, p<.001$, MSE $=265.98$, Rings Position, $F(5,160)=100.51, p<$ $.001, \mathrm{MSE}=126.41$, and a significant interaction effect, $F(10,320)=4.01, p<.001, \mathrm{MSE}=109.70$. The effect of Rings Position was explored further with a Tukey HSD test which revealed that performance in the $\mathrm{T} 2-1$ condition was higher than in the T2 -2 condition which was higher than in the remaining four conditions which did not differ from one another.

In broad outline, the results of the present experiment are in agreement with the results of Experiment 1. In this

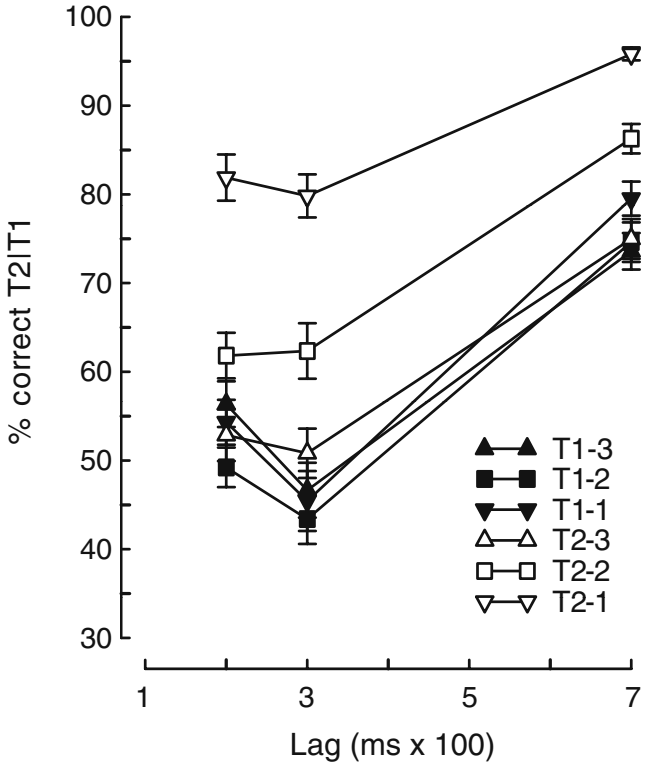

Fig. 8 Experiment 4. Percentage of correct identifications of the second target, given correct identification of the first target. The alerting stimulus was presented either three frames before the first target $(T 1-3)$ or two frames before the first target $(T 1-2)$ or in the frame preceding the first target $(T 1-1)$, or three frames before the second target $(T 2-3)$, or two frames before the second target (T2 $2)$, or in the frame preceding the second target $(T 2-1)$

respect, it is likely that, as was the case in Experiment 1, the interaction effect between Lag and Rings Position was due to the performance ceiling imposed by the response scale.

\section{Assessing the gradient of the strength of alerting}

The relationship between the strength of alerting and the temporal separation between the alerting stimulus and the trailing target is illustrated in Fig. 9, at each of the three lags, separately for $\mathrm{T} 1$ and $\mathrm{T} 2$. In every case, the functions reveal a clear gradient, with the strength of alerting diminishing as the temporal separation is increased beyond $100 \mathrm{~ms}$.

Statistical analysis of the data in Fig. 9 is subject to two considerations. First, interpretation of any interaction effects involving Rings Position is complicated by the possible limitation arising from the ceiling associated with the $100 \%$ limit of the response scale. Second, the number of rings positions ranged unsystematically between 3 and 6 . For these reasons, we opted to analyze only the results for T2 at Lag 3, which had the largest number of data points (see Fig. 7). These data were analyzed in a single-factor (Rings Position, $\mathrm{T}-6, \mathrm{~T}-5, \mathrm{~T}-4, \mathrm{~T}-3, \mathrm{~T}-2$, and $\mathrm{T}-1$ ) repeated-measures ANOVA. The analysis revealed that the effect of Rings Position was significant, $F(5,160)=48.45$, $p<.001$, MSE $=133.93$. This effect was explored further with a Tukey HSD test which revealed that performance in the $\mathrm{T}-1$ condition was higher than in the $\mathrm{T}-2$ condition 


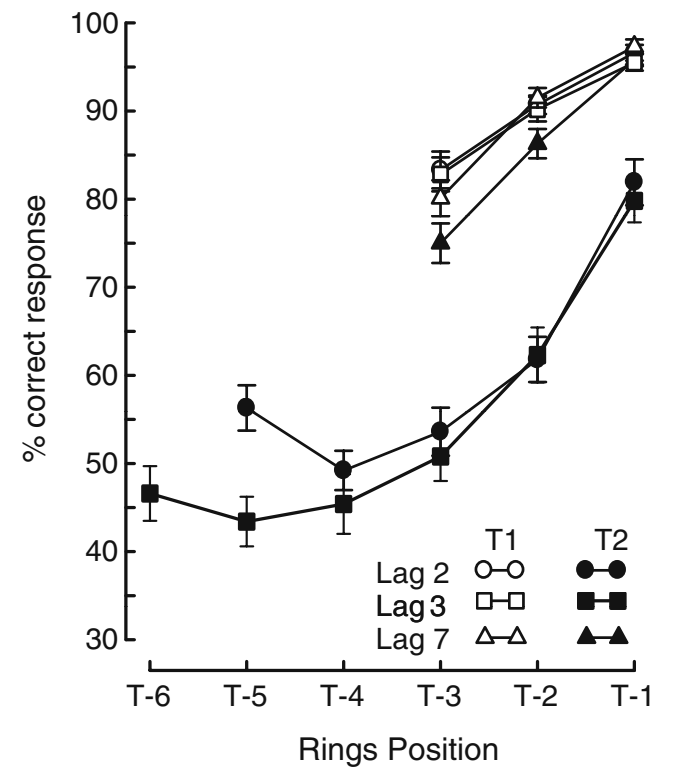

Fig. 9 Experiment 4. Percentage of correct identifications of the first target $(T 1)$ and the second target $(T 2)$, separately for Lags 2, 3, and 7

which was higher than in the remaining four conditions which did not differ from one another.

The strength of alerting clearly exhibits a temporal gradient that extends up to about $200 \mathrm{~ms}$. This estimate is consistent with Olivers and Van der Burg's (2008) finding that an accessory stimulus (a tone) presented $250 \mathrm{~ms}$ before $\mathrm{T} 2 \mathrm{had}$ no effect on identification accuracy of T2. This absence of an alerting effect in Olivers and Van der Burg's study is entirely consistent with the present finding that phasic alerting does not occur at lags beyond about $200 \mathrm{~ms}$ (Fig. 9). On the evidence in Fig. 9, the peak of the alerting function occurs when the alerting stimulus precedes the target by about $100 \mathrm{~ms}$. This estimate is in close agreement with the estimates reported by Nakayama and Mackeben (1989) who found that peak discrimination performance occurred when a transient cue was presented between 70 and $150 \mathrm{~ms}$ before the target.

\section{General discussion}

The present experiments examined the relationship between the $\mathrm{AB}$ and factors pertaining to the observer as distinct from factors external to the observer. In an earlier study, Olivers and Nieuwenhuis (2005) employed a tonic manipulation by inducing a relaxed attentional set in the observers - by rhythmic sounds or by pleasant memoriesand found the magnitude of the $A B$ to be reduced. The present work examined the effect of a phasic manipulation. Phasic changes in observer state were induced by presenting an alerting stimulus in temporal proximity to either T1 or T2 in an RSVP stream. We asked two related questions:
(1) whether the strength of the phasic response triggered by an alerting stimulus is reduced when the stimulus is presented during the period of the $\mathrm{AB}$, and (2) whether the magnitude of the $\mathrm{AB}$ is modulated by the presentation of an alerting stimulus.

Experiment 1 revealed that the presentation of an alerting stimulus led to a substantial improvement in identification accuracy of both T1 and T2. More important, in conjunction with Experiment 2, the results revealed that presenting the alerting stimulus during the period of the $\mathrm{AB}$ did not diminish the strength of the alerting effect. Conversely, the presence of an alerting stimulus, while enhancing the overall level of performance, did not affect the magnitude of the $\mathrm{AB}$, positively or negatively. Experiment 3 discounted the option that the improvement in performance might have arisen from the alerting stimulus acting as a predictive cue for the target. Finally, Experiment 4 revealed a steep temporal gradient of alerting that extended over a period of about $200 \mathrm{~ms}$ from the onset of the alerting stimulus.

This pattern of results is consistent with earlier findings that phasic changes in observer's state brought about by an alerting stimulus can boost performance substantially over a period of about $200 \mathrm{~ms}$ (Bernstein et al., 1970; Nakayama \& Mackeben, 1989; Schmidt et al., 1984). The result of principal interest to the present objectives, however, was that the phasic boost in performance combined additively with the $\mathrm{AB}$. More generally, it can be said that the $\mathrm{AB}$ and the phasic changes in observer state induced in the present study are orthogonal effects that neither enhance nor hinder one another.

Collectively, the results of the present experiments are consistent with the conclusion that alerting stimuli are processed without hindrance during the period of the $\mathrm{AB}$. This, as we noted above, is also true for briefly-presented spatial cues (Ghorashi et al., 2007, 2010). What causes these transient stimuli to remain unaffected even when presented while the system is busy processing T1? One plausible option is that the processing of brief transient stimuli-whether alerting stimuli or spatial cues - may take place along pathways that are separable from those involved in extracting the identity of the targets.

Distinct processing pathways for different classes of stimuli have been proposed in the context of both the $A B$ and visual search. To account for the outcome of a series of AB experiments, Spalek, Falcon, and Di Lollo (2006) proposed a hybrid input-filtering model in which endogenous and exogenous factors are subserved by different pathways. In that model, highly salient stimuli are said to gain direct access to high-level processing by bypassing an input filter set to exclude distractor items. Similarly, in a recent evolution of the Guided-Search model, Wolfe (2006) has proposed a model for visual-search performance 
consisting of two pathways. The Selective pathway is said to be dedicated to object-recognition processes and to be characterized by a bottleneck relatively early in the processing sequence. In contrast, the Nonselective pathway is said to be available for less demanding visual tasks that include image statistics and some aspects of scene analysis, and is deemed to be free from the early bottleneck.

The alerting stimuli used in the present work, and the spatial cues used in the experiments of Ghorashi et al. (2007, 2010), were invariably brief and were salient because the sudden mode of presentation produced distinctive onset transients within the visual system. It is plausible that, as was the case for Ghorashi et al.'s spatial cue, the processing of the attributes of suddenness and salience for the alerting stimulus, and the process of extracting the identity of T1, might have taken place in distinct pathways as proposed in the models of Spalek et al. (2006) and Wolfe (2006).

Beyond this obvious parallel, however, the present study differs from that of Ghorashi et al. (2007, 2010) in substantive and fundamental ways. Ghorashi et al. investigated the relationship between spatial pre-cuing and the AB. They were interested in whether the perception of a spatial pre-cue (a dot) presented at the exact location of the target was impaired during the AB. In contrast, no spatial pre-cuing was involved in the present work which used a spatially diffuse stimulus to examine the relationship between alerting and the AB. In brief, whereas Ghorashi et al. investigated the separability of exogenous spatial orienting and the $\mathrm{AB}$, we examined the separability of alerting and the $\mathrm{AB}$.

The finding that neither in the study of Ghorashi et al. $(2007,2010)$ nor in the present study was perception of the accessory stimulus (the spatial cue or the alerting stimulus) impaired during the period of the $\mathrm{AB}$ attests to the generality of the tenet that onset transients and identity extraction are processed along distinct pathways. That same tenet accounts for the present finding that the processing of an alerting stimulus neither interfered with-nor was interfered by - the processing of $\mathrm{T} 1$. On this view, a major function of the unhindered processing of the alerting stimulus was to set up the visual system in readiness for processing ensuing stimuli. Moreover, on the assumption that the alerting stimulus bypassed the early processing bottleneck, the alerting effect on target processing should be invariant with inter-target lag, as was the case in the present experiments.

\section{Theoretical considerations}

The independence of alerting and the $\mathrm{AB}$ demonstrated in the present work is not consistent with theories which stipulate that factors that influence the processing of $\mathrm{T} 1$, whether positively or negatively, should have corresponding effects on the AB. The resource-depletion account (e.g., Duncan et al., 1994), the bottleneck account, (e.g., Jolicœur \& Dell'Acqua, 1998), the delayed-attentional-engagement account (Nieuwenstein et al., 2005), and the temporary-lossof-control account (Di Lollo et al., 2005), all predict that to the extent that alerting speeds up the processing of $\mathrm{T} 1$, it should reduce the magnitude of the AB. Similarly disconfirmed is the locus-coeruleus account (Nieuwenhuis et al., 2005) which, as noted above, predicts a direct relationship between $\mathrm{T} 1$ alerting and the $\mathrm{AB}$.

A more successful account is provided by the Boost and Bounce (BB) model (Olivers \& Meeter (2008); see also Olivers, van der Stigchel, \& Hulleman, 2007, but at a cost. In this model, the AB is said to occur when the distractor presented directly after $\mathrm{T} 1$ accidentally passes an input filter set to pass only targets thus gaining access to highlevel processing. When this happens, the system is said to implement a temporary tightening of input control that causes trailing items - including T2 at the shorter lags - to be suppressed. As Olivers et al. pointedly note, the tightening of input control is triggered not by T1but by the first ensuing distractor. This means that facilitating the processing of T1by presenting an alerting stimulus, as was done in the present work, would not be expected to affect the magnitude of the AB. This prediction is supported by the independence between alerting and the $\mathrm{AB}$ found in the present work.

However, while avoiding the Scylla of independence of the $\mathrm{AB}$ and $\mathrm{T} 1$-performance, the $\mathrm{BB}$ hypothesis falls into the Charybdis of traditional findings. To wit, what the BB hypothesis cannot explain is why the magnitude of the $A B$ can vary inversely with the ease of $\mathrm{T} 1$ processing (Jolicœur, 1999; Visser, 2007) if it is the case that the AB is timelocked not to $\mathrm{T} 1$ but to the immediately ensuing distractor.

\section{The relationship between $\mathrm{T} 1$ processing and the $\mathrm{AB}$}

The present finding that enhanced $\mathrm{T} 1$ performance did not lead to the reduction in $\mathrm{AB}$ magnitude contrasts with the conventional finding that the magnitude of the $A B$ is related inversely to the ease of T1 processing (e.g., Jolicœur, 1999; Visser, 2007). A common account of this latter finding is that increasing the time taken to process T1 causes the processing of $\mathrm{T} 2$ to be delayed. Because, while delayed, the $\mathrm{T} 2$ representation is vulnerable to masking, the probability of T2 masking - and hence the magnitude of the $\mathrm{AB}$ - increases with the processing delay.

It is possible that these different outcomes might have arisen from the way in which the ease of T1 processing was manipulated. In the earlier studies, the ease of $\mathrm{T} 1$ processing was manipulated by means of tasks and stimuli that were plausibly processed along the same pathways as T1 itself. These included manipulations of sets size in visual 
search (Visser, 2007) and number of alternatives in an Nalternative forced-choice task (Jolicœur, 1999). In contrast, in the present work, $\mathrm{T} 1$ performance was manipulated by means of stimuli that, as noted above, may have been processed along separate pathways. This suggests that the inverse relationship between ease of $\mathrm{T} 1$ processing and $\mathrm{AB}$ magnitude might hold when ease of processing is manipulated by means of tasks and stimuli that are processed along the same pathways as T1, but not when they are processed along separate pathways.

On closer inspection, however, this option does not hold up. For theories that postulate an inverse relationship between ease of $\mathrm{T} 1$ processing and $\mathrm{AB}$ magnitude, what matters is not the way in which ease of processing is manipulated, but how soon T2 can gain access to high-level processing once $\mathrm{T} 1$ has been processed. In more general terms, the magnitude of the $\mathrm{AB}$ is said to be governed by factors that are time-locked to the completion of $\mathrm{T} 1$ processing. From this perspective, whether the alerting stimuli and $\mathrm{T} 1$ are processed along the same or different pathways would be largely irrelevant. For this reason, the present finding that alerting $\mathrm{T} 1 \mathrm{did}$ not lead to a decrement in $\mathrm{AB}$ magnitude cannot be explained by these theories in their current form.

Acknowledgments This work was supported by a Canadian Foundation for Innovation New Opportunities Grant and a grant from the British Columbia Knowledge Development Fund to T.M.S., and by Discovery Grants from the Natural Sciences and Engineering Research Council of Canada to T.M.S. and V.D.L.

\section{References}

Bernstein, I. H., Rose, R., \& Ashe, V. (1970). Preparatory state effects in intrasensory facilitation. Psychonomic Science, 19, 113-114.

Chun, M. M., \& Potter, M. C. (1995). A two-stage model for multiple target detection in rapid serial visual presentation. Journal of Experimental Psychology: Human Perception and Performance, 21, 109-127.

Di Lollo, V., Kawahara, J., Ghorashi, S. M. S., \& Enns, J. T. (2005). The attentional blink: Resource depletion or temporary loss of control? Psychological Research/Psychologische Forschung, 69, 191-200.

Duncan, J., Ward, R., \& Shapiro, K. L. (1994). Direct measurement of attentional dwell time in human vision. Nature, 369, 313-315.

Dux, P. E., Asplund, C. L., \& Marois, R. (2008). An attentional blink for sequentially presented targets: Evidence in favor of resource depletion accounts. Psychonomic Bulletin \& Review, 15, 809-813.

Fernandez-Duque, D., \& Posner, M. I. (1997). Relating the mechanisms of orienting and alerting. Neuropsychologia, 35, 477-486.

Ghorashi, S., Enns, J. T., Klein, R. M., \& Di Lollo, V. (2010). Spatial selection is separable from identity extraction in visual search. Journal of Vision, 0(0):1, 1-12, http://journalofvision.org/0/01/, doi:10.1167/0.0.1

Ghorashi, S. M., Klein, R. M., \& Di Lollo, V. (2007). Attentional orienting in response to peripheral cues survives the attentional blink. Visual Cognition, 15, 87-90.

Hebb, D. O. (1949). The organization of behavior. New York: Wiley.
Husain, M., Shapiro, K., Martin, J., \& Kennard, C. (1997). Abnormal temporal dynamics of visual attention in spatial neglect patients. Nature, 385, 154-156.

Jolicœur, P. (1999). Concurrent response-selection demands modulate the attentional blink. Journal of Experimental Psychology: Human Perception and Performance, 25, 1097-1113.

Jolicœur, P., \& Dell'Acqua, R. (1998). The demonstration of shortterm consolidation. Cognitive Psychology, 36, 138-202.

Kawahara, J., Di Lollo, V., \& Enns, J. T. (2001). Attentional requirements in visual detection and identification: Evidence from the attentional blink. Journal of Experimental Psychology: Human Perception and Performance, 27, 969-984.

Krope, K., Husain, M., \& Treue, S. (1998). An attentional blink for visual motion perception. Perception, 27(Suppl), 35c.

Lum, J. A. G., Conti-Ramsden, G., \& Lindell, A. K. (2007). The attentional blink reveals sluggish attentional shifting in adolescence with specific language impairment. Brain and Cognition, 63, 287-295.

Maciokas, J. B., \& Crognale, M. A. (2003). Cognitive and attentional changes with age: Evidence from attentional blink deficits. Experimental Aging Research, 29, 137-153.

Maki, W. S., Frigen, K., \& Paulson, K. (1997). Associative priming by targets and distractors during rapid serial visual presentation: Does word meaning survive the attentional blink? Journal of Experimental Psychology: Human Perception and Performance, 23, 1014-1034.

Mason, D. J., Humphreys, G. W., \& Kent, L. (2005). Insights into the control of attentional set in ADHD using the attentional blink paradigm. Journal of Child Psychology and Psychiatry, 46, $1345-1353$.

Nakayama, K., \& Mackeben, M. (1989). Sustained and transient components of focal visual attention. Vision Research, 29, 16311647.

Nieuwenhuis, S., Gilzenrat, M. S., Holmes, B. D., \& Cohen, J. D. (2005). The role of the locux Coeruleus in mediating the attentional blink: A neurocomputational theory. Journal of Experimental Psychology: General, 134, 291-307.

Nieuwenstein, M. R., Chun, M. M., van der Lubbe, R. H. J., \& Hooge, I. T. C. (2005). Delayed attentional engagement in the attentional blink. Journal of Experimental Psychology: Human Perception and Performance, 31, 1463-1475.

Olivers, C. N. L. (2004). Blink and shrink: The effect of the attentional blink on spatial processing. Journal of Experimental Psychology: Human Perception and Performance, 30, 613-631.

Olivers, C. N. L., \& Meeter, M. (2008). A boost and bounce theory of temporal attention. Psychological Review, 115, 836-863.

Olivers, C. N. L., \& Nieuwenhuis, S. (2005). The beneficial effect of concurrent task-irrelevant mental activity on temporal attention. Psychological Science, 16, 265-269.

Olivers, C. N. L., Spalek, T. M., Kawahara, J., \& Di Lollo, V. (2009). The attentional blink: Increasing target salience provides no evidence for resource depletion. A commentary on Dux, Asplund, and Marois (2008). Psychonomic Bulletin \& Review, 16, 214-218.

Olivers, C. N. L., \& Van der Burg, E. (2008). Bleeping you out of the blink: Sound saves vision from oblivion. Brain Research, 1242, 191-199.

Olivers, C. N. L., van der Stigchel, S., \& Hulleman, J. (2007). Spreading the sparing: Against a limited-capacity account of the attentional blink. Psychological Research, 71, 126-139.

Raymond, J. E., Shapiro, K. L., \& Arnell, K. M. (1992). Temporary suppression of visual processing in an RSVP task: An attentional blink? Journal of Experimental Psychology: Human Perception and Performance, 18, 849-860.

Rolke, B., Heil, M., Streb, J., \& Hennighausen, E. (2001). Missed prime words within the attentional blink evoke an N400 semantic priming effect. Psychophysiology, 38, 165-174. 
Ross, N. E., \& Jolicœur, P. (1999). Attentional blink for color. Journal of Experimental Psychology: Human Perception and Performance, 25, 1483-1494.

Schmidt, R. A., Gielen, S. C., \& van den Heuvel, P. J. (1984). The locus of intrasensory facilitation of reaction time. Acta Psychologica, 57, 145-164.

Shapiro, K. L., Arnell, K. M., \& Drake, S. H. (1991). Stimulus complexity mediates target detection in visual attention search [Abstract]. Investigative Ophthalmology and Visual Science, 32 (Suppl.), 1040.

Shapiro, K. L., Caldwell, J., \& Sorensen, R. E. (1997). Personal names and the attentional blink: A visual "cocktail party" effect. Journal of Experimental Psychology: Human Perception and Performance, 23, 504-514.

Shapiro, K. L., Driver, J., Ward, R., \& Sorensen, R. E. (1997). Priming from the attentional blink: A failure to extract visual tokens but not visual types. Psychological Science, 8 , 95-100.

Shapiro, K. L., Raymond, J. E., \& Arnell, K. M. (1994). Attention to visual pattern information produces the attentional blink in RSVP. Journal of Experimental Psychology: Human Perception and Performance, 20, 357-371.
Shapiro, K. L., Schmitz, F., Martens, S., Hommel, B., \& Schnitzler, A. (2006). Resource sharing in the attentional blink. NeuroReport, 17, 163-166.

Spalek, T. M., Falcon, L. J., \& Di Lollo, V. (2006). Attentional blink and attentional capture: Endogenous versus exogenous control over paying attention to two important events in close succession. Perception \& Psychophysics, 68, 674-684.

Visser, T. A. W. (2007). Masking T1 difficulty: Processing time and the attentional blink. Journal of Experimental Psychology: Human Perception and Performance, 33, 285-297.

Visser, T. A. W., Bischof, W. F., \& Di Lollo, V. (1999). Attentional switching in spatial and non-spatial domains: Evidence from the attentional blink. Psychological Bulletin, 125, 458-469.

Vogel, E. K., Luck, S. J., \& Shapiro, K. L. (1998). Electrophysiological evidence for a postperceptual locus of suppression during the attentional blink. Journal of Experimental Psychology: Human Perception and Performance, 24, 1656-1674.

Ward, R., Duncan, J., \& Shapiro, K. (1996). The slow time-course of visual attention. Cognitive Psychology, 30, 79-109.

Wolfe, J. M. (2006). Guided Search 4.0: Current progress with a model of visual search. In W. Gray (Ed.), Integrated models of cognitive systems (pp 99-119). New York: Oxford. 